\title{
Chiral nanomaterials for tumor therapy: autophagy, apoptosis, and photothermal ablation
}

\author{
Zaihui Peng ${ }^{1}$, Long Yuan ${ }^{1}$, Juncheng XuHong ${ }^{1}$, Hao Tian ${ }^{1}$, Yi Zhang ${ }^{1 *}$, Jun Deng ${ }^{2^{*}}$ (D) and Xiaowei Qi ${ }^{*}$
}

\begin{abstract}
Chirality is a fundamental characteristic of natural molecules and a crucial factor in the biochemical reactions of living cells and organisms. Recently, researchers have successfully introduced chiral molecules to the surfaces of nanomaterials, creating chiral nanomaterials that exhibit an upscaling of chiral behavior from the molecular scale to the nanoscale. These chiral nanomaterials can selectively induce autophagy, apoptosis, and photothermal ablation in tumor cells based on their chirality, making them promising for application in anti-tumor therapy. However, these interesting and important phenomena have hitherto received little attention. Accordingly, we herein present a review of recent research progress in the field of chiral nanomaterials for tumor therapy along with brief looks at the mechanistic details of their actions. Finally, the current challenges and future perspectives of chiral nanomaterials in terms of maximizing their potential in tumor therapy are discussed. Thus, this review provides a helpful introduction to the design of chiral nanomaterials and will hopefully highlight the importance of chirality in tumor therapy.
\end{abstract}

Keywords: Chirality, Nanomedicine, Biomaterials, Tumor therapy

\section{Introduction}

Chirality is a crucial property of natural molecules, chiral isomers have specific and selective effects on biological systems [1], which has extraordinary significance for a variety of biological events, including cell metabolism, cell fate, and even the evolution of organisms [2, 3]. For instance, in Eukarya, D-nucleotides, L-amino acids and L-phospholipids are homochiral building blocks from which live organisms are formed [4-6], while for bacterial, D-amino acids, i.e., D-Ala and D-Glu, present in the peptidoglycan on bacterial cell wall that act to provide

*Correspondence: ZY53810@163.com; djun.123@163.com; qxw9908@foxmail.com

†Zaihui Peng and Long Yuan contributed equally to this work.

${ }^{1}$ Department of Breast Surgery, Southwest Hospital, Army Medical University, Chongqing 400038, China

2 Institute of Burn Research, Southwest Hospital, State Key Lab of Trauma, Burn and Combined Injury, Army Medical University, Chongqing 400038, China resistance to most known proteases. Nanoparticles (NPs) have unique size and surface properties, which can enhance or expand their chiral effects $[7,8]$. The introduction of molecular chirality into the surface of nanomaterials provides a new type of biological material-chiral nanomaterials. This new type of nanoscale chiral materials realizes a scale-leap for chiral signals from the molecular scale to the nanoscale, and exhibits superior properties to those of ordinary achiral nanomaterials $[8,9]$.

Related studies have found that the pharmacological activities of different enantiomers of chiral drugs sometimes show no obvious differences, but sometimes they may cause different or even opposite therapeutic effects. For example, in the antiarrhythmic effect of propafenone, its two isomers have similar pharmacological activities [10]. However, for the two enantiomers of propoxyphene, L-propoxyphene is a cough suppressant and D-propoxyphene is an analgesic. original author(s) and the source, provide a link to the Creative Commons licence, and indicate if changes were made. The images or other third party material in this article are included in the article's Creative Commons licence, unless indicated otherwise in a credit line to the material. If material is not included in the article's Creative Commons licence and your intended use is not permitted by statutory regulation or exceeds the permitted use, you will need to obtain permission directly from the copyright holder. To view a copy of this licence, visit http://creativecommons.org/licenses/by/4.0/. The Creative Commons Public Domain Dedication waiver (http://creativeco mmons.org/publicdomain/zero/1.0/) applies to the data made available in this article, unless otherwise stated in a credit line to the data. 
The two exhibit completely different pharmacological activities [11]. D-/L-dopamine is a drug used for the treatment of Parkinson's disease, but L-dopamine is therapeutically active, while D-dopamine can cause harm if it accumulates in the body [12]. Furthermore, the R-enantiomer of thalidomide has a therapeutic effect, while the S-enantiomer has a strong teratogenic effect [13]. The fascinating functions of chiral materials inspires tremendous amounts of research on developing functional chiral medicine [14]. For instance, chiral noble-metal NPs and quantum dots (QDs) that exhibit enantioselective catalytic properties and wide substrate generality have been reported $[15,16]$. Furthermore, the interactions between nanomaterials and biological systems and their influence on phenomena such as cellular uptake and elimination have been widely studied as a means to further the application of chiral nanomaterials in biomedicine and bioengineering and to ensure their safety [17]. Moreover, chirality-based optical sensing and therapy strategies are becoming increasingly common [18-20]. The current applications of chiral nanomaterials are illustrated in Fig. 1.

Cancer is the greatest threat to human health and is becoming more serious because of factors such as environmental pollution and unhealthy lifestyle choices. Therapeutic strategies for clinical cancer treatment are dominated by traditional therapies involving surgery, chemotherapy, and/or radiotherapy. However, these traditional therapies are subject to several problematic factors [21-23] involving recurrence and secondary metastasis, poor tumor targeting, drug resistance,

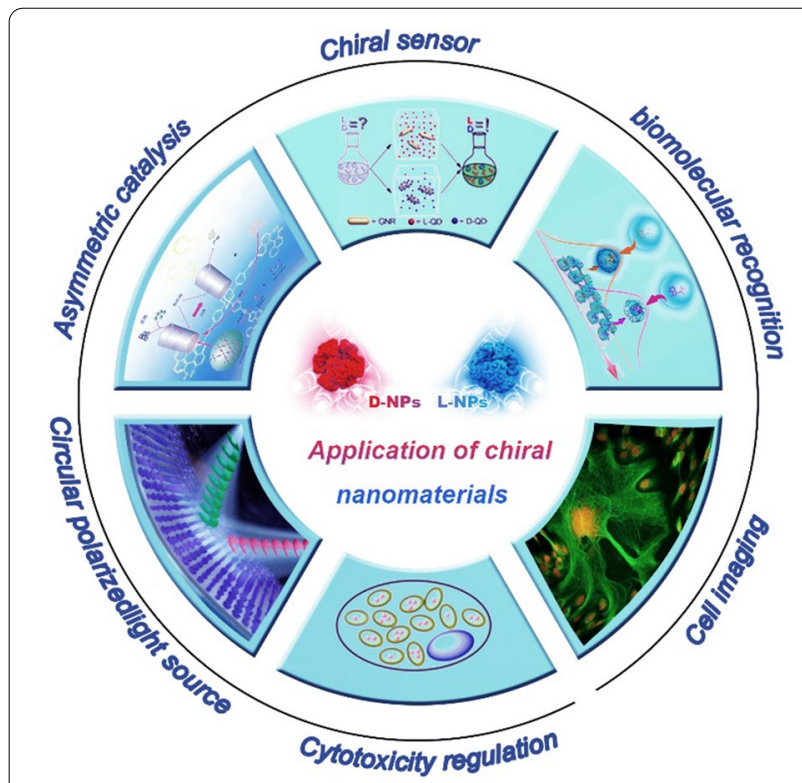

Fig. 1 Some current applications of chiral nanomaterials and radiation tolerance. Thus, strategies for enhancing the therapeutic effects and overcoming the problems of traditional therapies have been developed [2427]. One such promising strategy is the use of chiral nanomedicine.

Many chiral nanomaterials have been fabricated for anti-tumor therapy. These materials can induce autophagy and apoptosis of tumor cells with high specificity and efficiency by virtue of the unique size and surface properties of NPs and the unique selectivity of the organism's chiral enantiomers [7, 8]. For example, chiral glutathione $(\mathrm{GSH})$-modified cadmium telluride $(\mathrm{CdTe})$ QDs have been demonstrated to produce a large number of autophagic vacuoles in tumor cells and activate autophagy in a chirality-dependent way, where L-GSHQDs induce more intense autophagy than D-GSH-QDs [28]. Furthermore, $\mathrm{Du}$ et al. modified the surfaces of NPs with L/D phosphotyrosine to synthesize chiral NPs (NP@-L/D-PYs). The alkaline phosphatase on the surface of cancer cells dephosphorylates the NP@-D-PYs, enabling them to adhere strongly to cancer cells, where they can activate exogenous cell death pathways and induce apoptosis. Interestingly, the NP@-L-PYs have almost no effect on cancer cells.

After the introduction of chiral amino acid ligands, a large number of amine groups and carboxyl groups are presented on the surfaces of the NPs, imparting excellent water solubility and suitable chemically reactive groups [29]. The chemically reactive groups attached to their surfaces being able to adopt a range of structural configurations, thus allowing electronic structures with different energy levels. This enhances the possibility for the recombination of electrons and holes captured by the surface states [30], which increases the fluorescence quantum yield of chiral NPs, which in turn makes them have excellent photothermal properties [31, 32]. Make chiral nanomaterials have broad application prospects in photothermal tumor treatment. For example, Li et al. used chiral cysteine to reduce $\mathrm{MoO}_{3}$, obtaining a dual visible- and near-infrared (NIR)-light-active nanomaterial (L/D-Cys- $\mathrm{MO}_{3-\mathrm{x}} \mathrm{NPs}$ ) with strong chiral effects. After being endocytosed by tumor cells, the NPs generate temperatures in the range $40-50{ }^{\circ} \mathrm{C}$ upon absorbing circularly polarized light (CPL), thereby inducing cell death by denaturing intracellular proteins and rupturing cell membranes. L/D-Cys- $\mathrm{MO}_{3-\mathrm{X}} \mathrm{NPs}$ exhibit a higher photothermal conversion efficiency and tumor-cell-killing effect than traditional hyperthermic molybdenum oxide [33].

A considerable number of studies on the application of chiral ligands into nanomaterials in cancer therapy have been reported. Nevertheless, many questions remain to be answered. For example, do D or L chiral nanomaterials 
have better antitumor effects, and what are the mechanisms of their actions? Clearly, an informed summary of the biological functions and mechanisms of chirality in tumor therapy is needed. Accordingly, in this review, we consider the autophagy, apoptosis, and photothermal ablation of the three important types of tumor cells death as induced by chiral nanomaterials in separate sections. Then, the potential mechanisms by which chiral nanomaterials regulate tumor cells is briefly introduced. Finally, the future perspectives of chiral nanomaterials in terms of challenges currently faced in the field and some strategies to overcome them are presented. Overall, we hope to provide a theoretical basis and reference source for the development and application of new chiral nanomaterials in tumor therapy.

\section{Chiral nanomaterials that induce autophagy in tumor cells}

Autophagy is a lysosome-based catabolic process that maintains homeostasis and the defense capabilities of cells by removing damaged and/or redundant proteins and organelles. Usually, after autophagy, endogenous and foreign substances are held in vesicles until they are finally degraded by lysosomes [34]. A series of studies have shown that autophagy has a close and complex relationship with the development and progression of tumors. For instance, autophagy can enhance the tolerance of tumor cells to tissue hypoxia, acidosis, and other adverse environments. Conversely, autophagy can eliminate damaged proteins and organelles to avoid genome damage, thereby suppressing the development of tumors. It is currently believed that during tumorigenesis, autophagy mainly inhibits the development and progression of tumor cells [35]. Thus, controlling the autophagy level of tumor cells may provide a promising strategy for the effective treatment of malignant tumors [36, 37], especially that of autophagy-deficient cancers, such breast, ovarian, and prostate cancers [38]. For instance, adjusting autophagy can change the sensitivity of triplenegative breast cancer cells to radiotherapy and reduce their resistance to chemotherapeutic drugs [39].

The features of chiral NPs, such as composition, size, shape, and surface functionalization, can regulate autophagy by inducing intracellular oxidative stress or changing the expression of autophagy-related genes/proteins [40-42]. Furthermore, recent related research has revealed that NPs as foreign materials in cells can influence autophagy [43].

The chirality-dependences of cytotoxicity [44], cell differentiation [45, 46], cellular uptake [47], cell adhesion [48], and protein adsorption $[49,50]$ are widely accepted. Accordingly, NPs bearing surface-anchored chiral molecules have been widely demonstrated to modulate autophagy in tumor cells. For instance, in 2017, Hu's group used natural chiral lysine to synthesize a chiral polymer with a molecular weight of $\sim 5911 \mathrm{Da}$ (Fig. 2a), that forms as unique L/D peptide dendrimers (L/D-PNs) with about $8 \mathrm{~nm}$ (Fig. 2b). In addition, the stereochemical properties of homochiral PNs were studied using optical rotation research (Fig. 2c, d). The L/D-PNs quickly activate the autophagy of tumor cells, inducing the formation of autophagosomes and autolysosomes (Fig. 2e). Moreover, this autophagy induction shows chirality dependence, with that by the D-type dendrimers being stronger than that by the L-type [51].

In 2018, Sun and coworkers used DNA self-assembly technology to assemble gold NPs (AuNPs) with coreshell structures into a chiral tetrahedral structure. One of the DNA ends was modified with a polypeptide chain that could be hydrolyzed using the autophagy marker ATG4b, and up-conversion NPs (UCNPs) were further assembled in the tetrahedron using ATP aptamers. Modification of $\mathrm{D} / \mathrm{L}$-glutathione on the surface of the assembly enabled the tetrahedron to present tunable circular dichroism (CD). When tetrahedrons with embedded UCNPs are cultured with cancer cells, the D-glutathione-modified tetrahedron structure causes the cells to produce a strong autophagy response, and this autophagy process can be monitored in real time through $\mathrm{CD}$ and up-conversion luminescence measurement, as illustrated in Fig. 3 [52].

Wang et al. reported the fabrication of $\mathrm{D}$ - and L-cysteine-modified $\mathrm{Cu}_{2-\mathrm{x}} \mathrm{S}$ nanocrystals (NCs) using a sacrifice template-ligand exchange strategy. Their interactions with cells and ability to induce tumor cell autophagy as well as their photothermal ablation properties were investigated. The authors reported that the NCs induce the production of large amounts of reactive oxygen species (ROS) in tumor cells, which promotes cellular autophagy. Furthermore, the cell ablation is improved by the photothermal effects of the $\mathrm{Cu}_{2-\mathrm{x}} \mathrm{S} \mathrm{NCs}$, and the autophagy activation was found to be chirality dependent, with $\mathrm{D}-\mathrm{Cu}_{2-\mathrm{x}} \mathrm{S}$ NCs causing more extensive autophagy than $\mathrm{L}-\mathrm{Cu}_{2-\mathrm{x}} \mathrm{S} \mathrm{NCs}$ [53].

Interestingly, and as is inconsistent with the results of the above study, Li et al. attached chiral GSH to the surface of CdTe QDs to obtain chiral QDs (L/D-GSH-QDs), as illustrated in Fig. 4a. Transmission electron microscopy (TEM) indicated that all QDs are approximately spherical in shape. The lattice spacing of the (220) plane for D560 and L563 QDs showed a distance of $0.23 \mathrm{~nm}$, and of the (111) plane for D622 and L620 QDs a distance of $0.38 \mathrm{~nm}$ (Fig. 4b). The chiral QDs were co-cultured with human liver cancer cells, revealing that both L- and D-GSH-QDs exhibit dose-dependent cytotoxicity and significantly increase the levels of autophagic vacuoles. However, the autophagy activation was found to be 


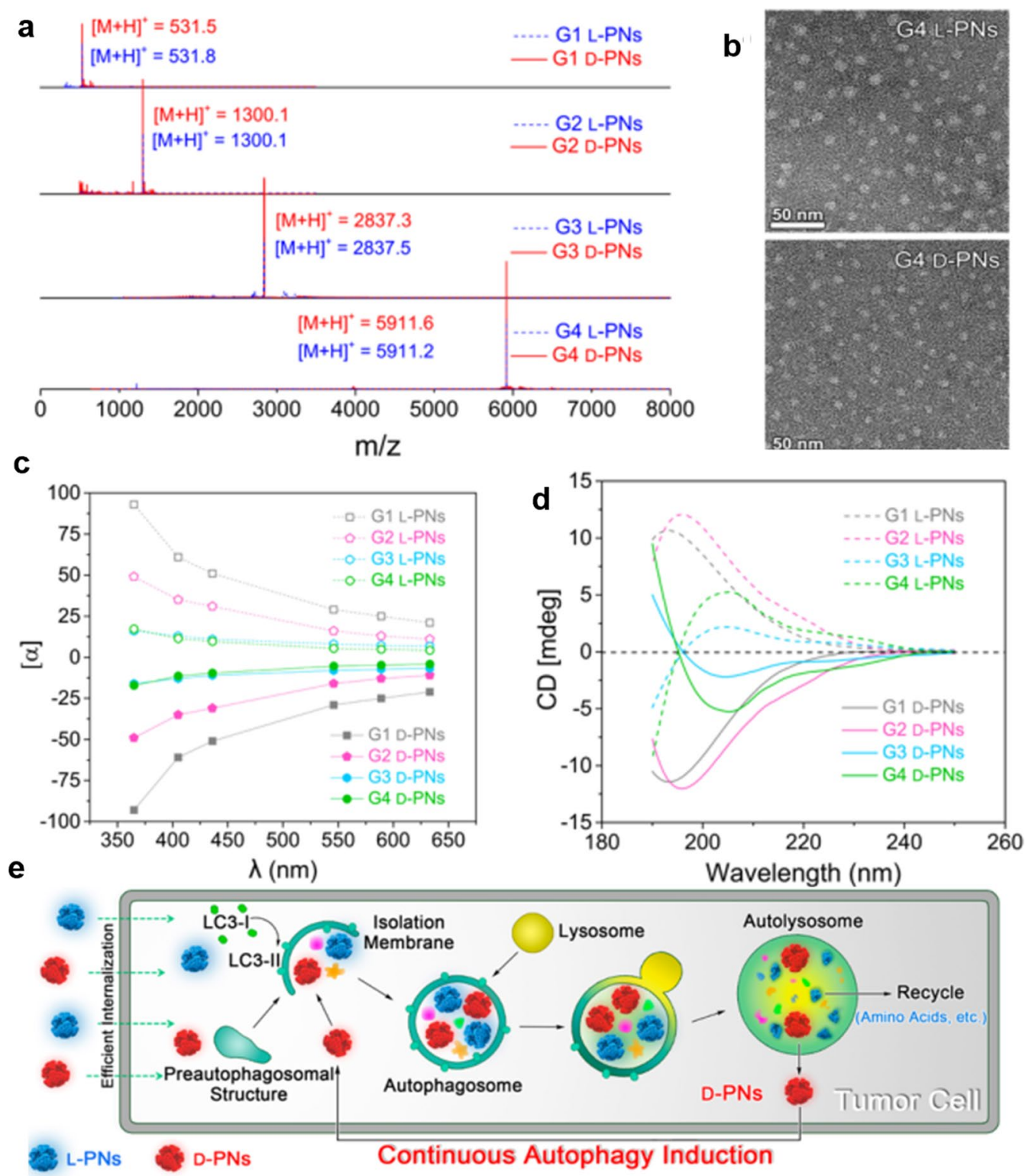

Fig. 2 Characterization data for chiral L/D-PN protein nanomimics and a schematic of their effect upon autophagy processes and mechanisms. a Mass spectra; $\mathbf{b}$ transmission electron microscopy images; c optical rotatory dispersion curves; and $\mathbf{d}$ circular dichroism spectra. e Schematic of the influence of L/D-PNs upon autophagy processes. Reprinted with permission from ref. [51]. Copyright (2017) American Chemical Society

chirality-dependent, with L-GSH-QDs being the more effective (Fig. 4c, d) [28].

Xin et al. synthesized novel chiral zinc-aspartate nanofibers (L/D-(Zn-ASP) NFs) that were found to be over $30 \mu \mathrm{m}$ in length and highly uniform with diameters $<100 \mathrm{~nm}$ by an interfacial polymerization reaction between L/D aspartate and zinc ions (Fig. $5 \mathrm{a}-\mathrm{c}$ ). The polymer retained in the extracellular environment can strongly interact with eHSP90 from the cancer cell, which induces decrease in the level of gelatinases, resulting in downregulation of nuclear factor-kappa B (NF-kB) signaling and formation of autophagy, finally inhibiting cancer cell proliferation, migration, and invasion (Fig. 5d) [54].

Research on chiral dependence in autophagy induced by nanomaterials is still at an early stage and there are many controversies. To explore this topic, our group synthesized AuNPs coated with the L, D, and racemic forms of chiral poly (acryloyl valine) to form L-PAV-AuNPs, D-PAV-AuNPs, and L/D-PAV-AuNPs, respectively 

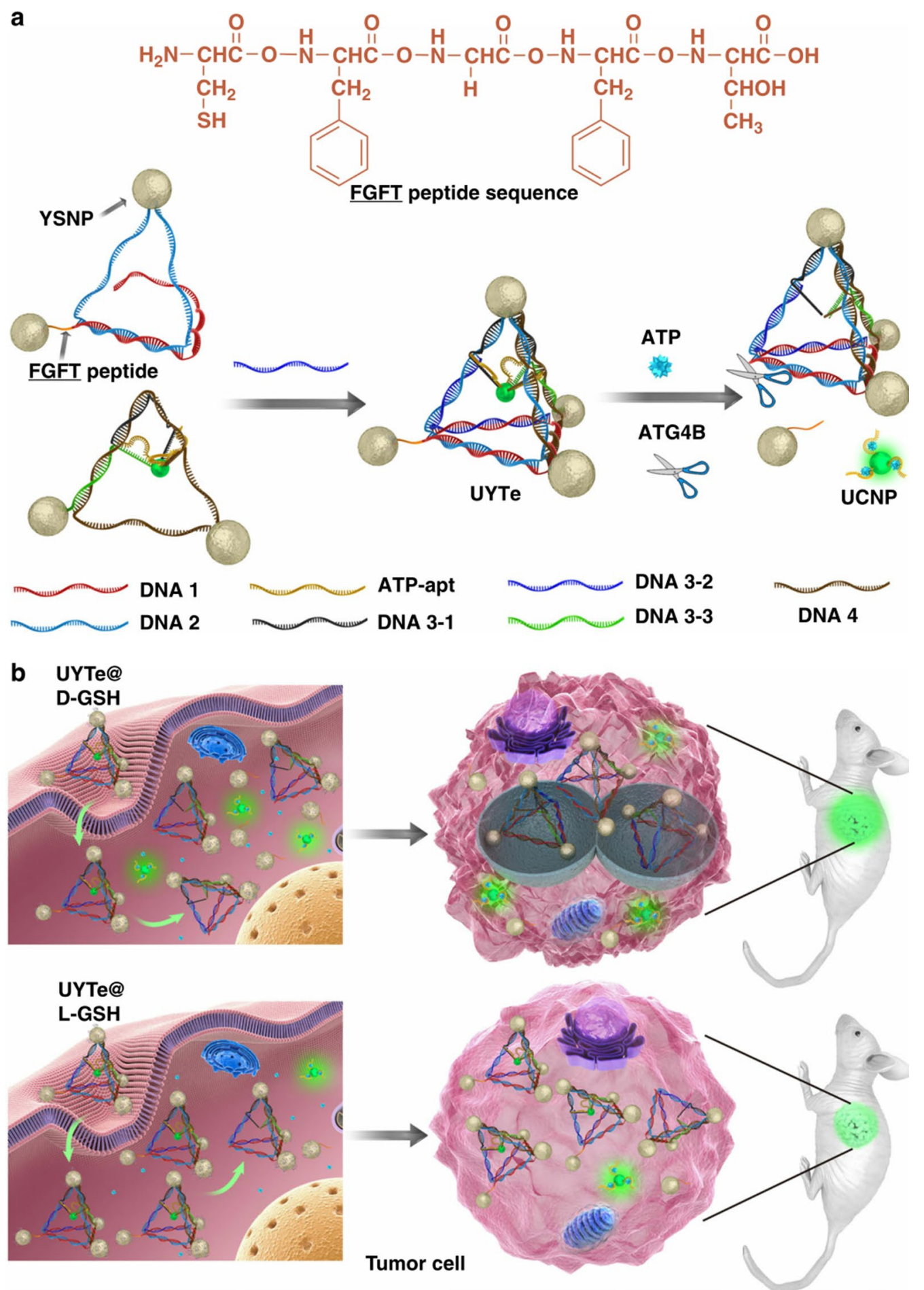

Fig. 3 Schematic of a chiral nanodevice for autophagy induction and observation. a self-assembly of UCNP-centered yolk-shell tetrahedrons, and $\mathbf{b}$ their use to detect autophagy and ATP. Reprinted with permission from ref. [52]. Copyright (2018) Springer Nature

(Fig. 6a). We found that L(D)-PAV-AuNPs induce ROS and lysosome function, thereby causing cells to undergo autophagy and cell death, in a chirality-dependent manner. The inhibitory effect of D-PAV-AuNPs was found to be stronger than that of the L form (Fig. 6b). Additionally, L(D)-PAV-AuNPs show very low toxicity to normal 3T3 fibroblasts and breast epithelial cells, have the ability 
a
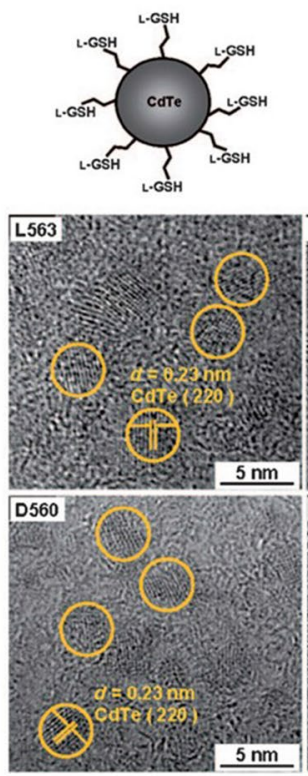
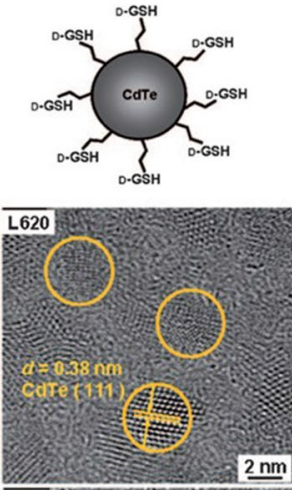

D622

$2 \mathrm{~nm}$

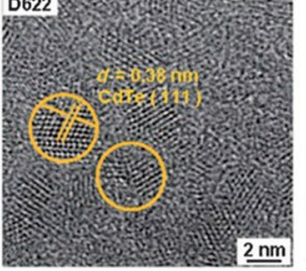

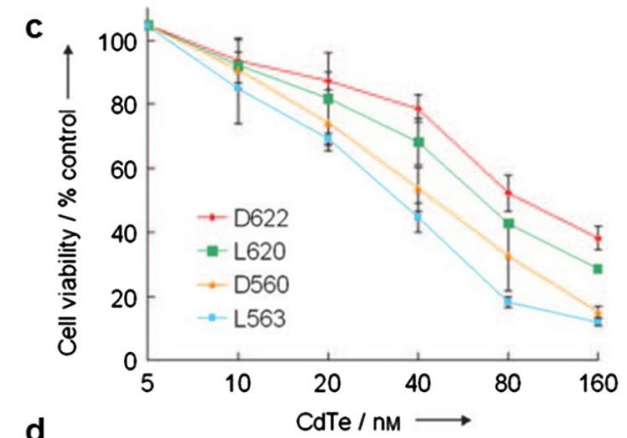

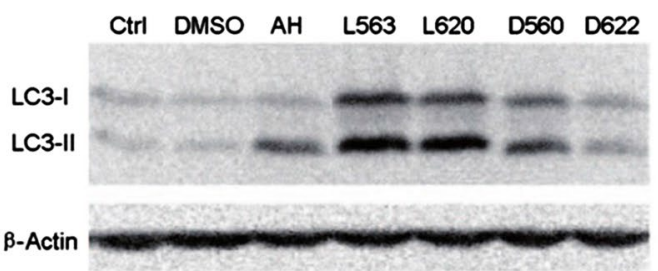

Fig. 4 a Schematic structures of L- (left) and D-GSH-QDs (right). b High-resolution transmission electron microscopy images of D- and L-GSH-QDs. The circles indicate the spacings of the CdTe (220) or (111) lattices. c Chirality- and concentration-dependent cytotoxicities of the QDs. $\mathbf{d}$ L-GSH-QDs induce autophagy more effectively than D-GSH-QDs (40 nm). Reprinted with permission from ref. [28]. Copyright 2011 WILEY-VCH Verlag GmbH \& Co. KGaA, Weinheim

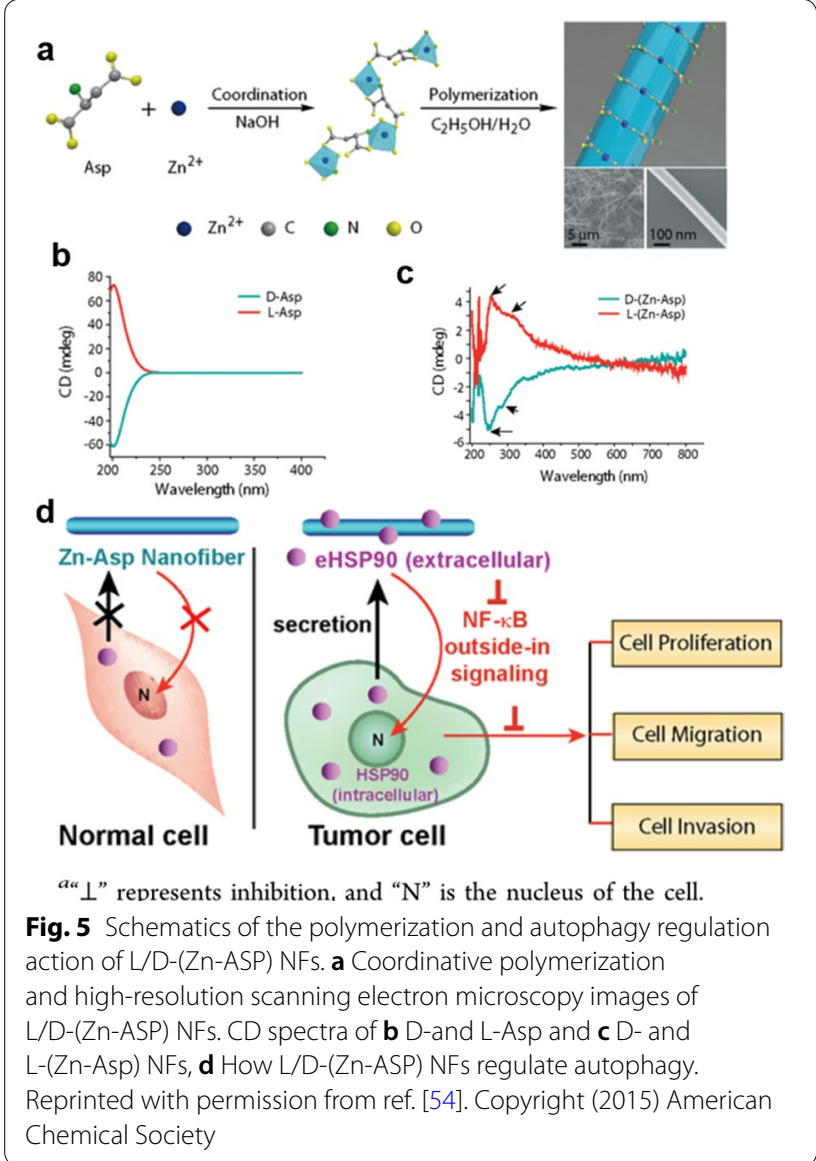

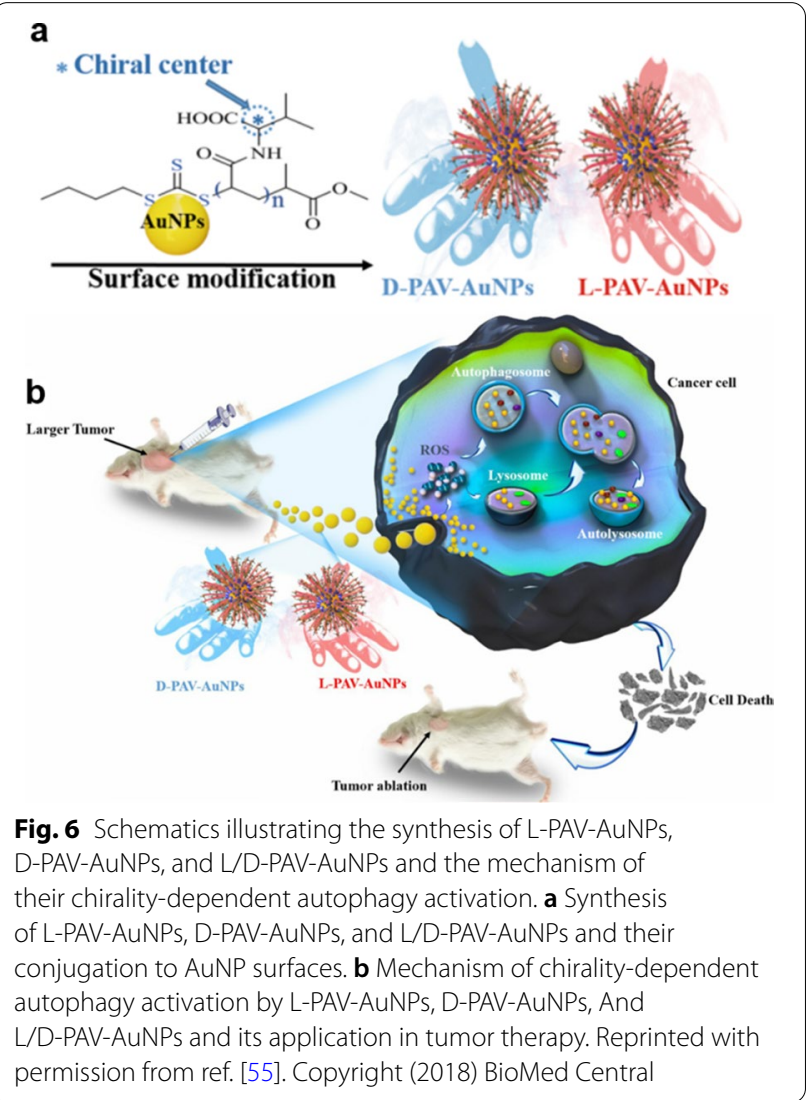


to induce tumor cells with high selectivity, and thus have great potential for biological application [55].

The above studies show that chiral nanomaterials that induce autophagy in tumor cells do so in a chiralitydependent manner, with the dextrorotatory-polymermodified nanomaterials having a stronger autophagy induction effect. This phenomenon may be due to the main way for NPs to enter cells is by micropinocytosis [47]. After L/D-NPs enter the cell, the L-phospholipid molecular layer on the surface of the cancer cell is selectively more inclined to combine with heteroenantiomeric polymers (D-NPs). As a result, regardless of the concentration of chiral NPs, D-NPs show greater absorption than L-NPs [52]. As D-NPs accumulate, more ATP will be produced by the reaction of NPs with biological macromolecules in the cell [4], and autophagy-related reactions depend on the expression of ATP [56, 57]. D-NPs accumulate more, and the interaction with cell surface receptors induces more ROS [55], ROS are necessary for inducing autophagy [58], and thus induce the autophagy of cancer cells to a greater extent. Secondly, the biological activities and metabolism of endogenous proteins are regulated by chirality-specific enzymes. Compared with L-NPs, D-NPs are not easily degraded by natural proteases and coenzymes in the cellular environment. It may be precisely because D-NPs are tolerant to biodegradation that they can more continuously and efficiently induce autophagy $[33,59]$. In addition, the amino acid moiety of the chiral NPs could form hydrogen bond with the specific protein secreted by cancer cells, which might enhance the binding interaction [60], and the intensity of the hydrogen bonds between chiral NPs and specific protein can be different considering the different spatial arrangements of the functional groups (the uncoordinated carboxyl and amine group of chiral Asp) on the surface of the two kinds of NPs[61]. Therefore, the binding between D-/L-NPs and specific protein are not exactly the same, resulting in a slight difference in their autophagy ability toward cancer cells.

Amino acids have been widely used for studying the interaction between cells and chiral surface due to their versatility and biocompatibility [62], and amino type could influence the chiral effects on biological systems [63]. Interestingly, in the studies discussed above, Sun et al. [52] and $\mathrm{Li}$ et al. [28] both used glutathione for chiral NP fabrication, but their results for the chiralitydependence of autophagy induction in cancer cells are completely opposite. This may be because the former mainly benefits from the stronger uptake of D-type NP by cells, while the abundance of L-GSH and relative scarcity of D-GSH in biological systems may lead to differences in the shell degradation behavior of the QDs. GSH-coated QDs will more readily interact with GSH of the same enantiomer. This difference makes the thermodynamic stability of QDs L-chiral dependent.

These results provide insights for the development of new chiral NPs that induce autophagy in tumor cells in a chirality-dependent manner. However, it should be noted that the complexity and heterogeneity of biomolecules and cells mean that many questions have yet to be answered and ongoing research must be performed.

\section{Chiral nanomaterials that induce apoptosis in tumor cells}

Apoptosis, also known as programmed cell death, is a physiological process involving multiple factors, including the immune response, gene regulation, and signal transduction [64]. Apoptosis plays a very important regulatory role in a variety of normal physiological processes. Thus, abnormal apoptosis can give rise to a range of pathological effects, such as tumors, autoimmune diseases, and neurodegenerative diseases [65]. Studies in recent years have confirmed that the unregulated growth of tumor cells is the result of inhibited tumor cell apoptosis. Therefore, apoptosis disorders are closely related to tumor occurrence, development, and regression [66]. Accordingly, several scholars believe that inducing apoptosis in tumor cells may become a viable means of treating cancer [67]. Furthermore, recent studies have demonstrated that NPs can activate caspase-family proteins as well as the expression of DNA-damage-repair proteins and pro-apoptotic gene P53, thus promoting cancer cell apoptosis [68-70].

The functionality of a biological system is entirely dependent on the physical structures of its components. This means that, in chirality-specific biological interactions, one enantiomer of a molecule can have an important biological function while the other may be inactive or even harmful. Thus, numerous researchers have applied chiral nanotechnology to induce apoptosis in cancer cells.

Du et al. decorated iron oxide NPs with D/L-phosphotyrosine to form bioactive magnetic NPs (NP@D/L-pYs) with diameters of $\sim 10 \mathrm{~nm}$. These NPs cluster somewhat to form small aggregates with an average diameter of $500 \mathrm{~nm}$ (Fig. 7a). When cancer cells are co-cultured with the NPs, the alkaline phosphatase on the surface of the cancer cells dephosphorylates the pY moieties, allowing NP@-D-Y to strongly adhere to the cancer cells and activate exogenous cell death, causing apoptosis (Fig. 7b) [71].

In 2019, Yeom et al. assembled chirality-engineered supraparticles (SPs) from small chiral CdTe NPs and modified their surfaces with chiral cysteine to synthesize ultrafine chiral SPs (L/D-SPs) (Fig. 8a). The ultrafine 


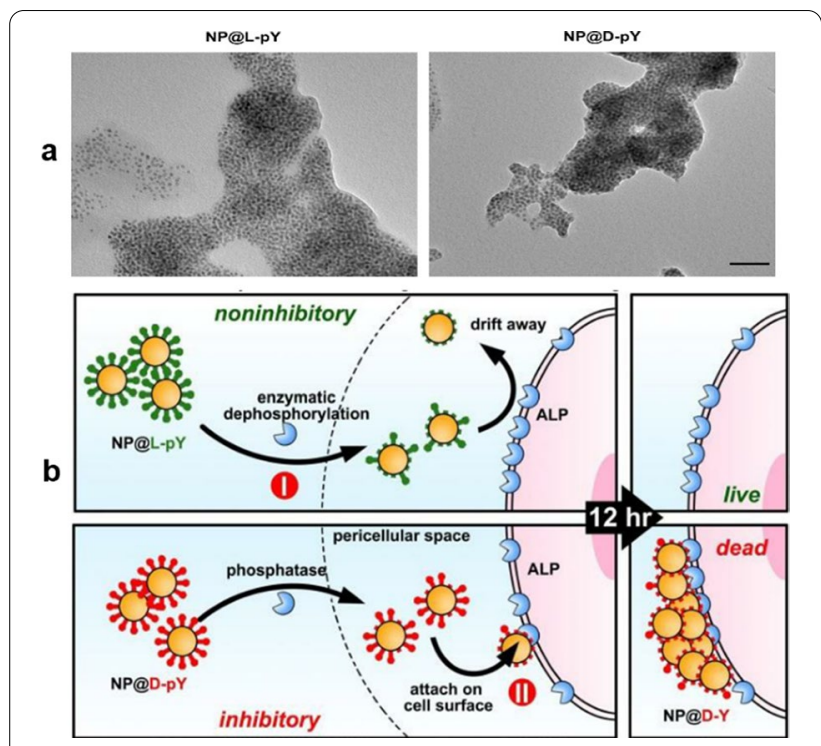

Fig. 7 Chiral-NP-controlled reaction-diffusion leads to cell death. a Transmission electron microscopy images of NP@L-pYs (left), and NP@D-pYs (right). Scale bar=50 nm. b Enzymes in the culture medium dephosphorylate the pY groups on the NP@L-pYs but not those on the NP@D-pYs. Then, the alkaline phosphatases on the cancer cell surfaces dephosphorylate most of the pYs remaining on the NP@D-pYs, causing them to adhere to the cells and evoke apoptosis. Reprinted with permission from ref. [71]. Copyright (2017) Wiley-VCH Verlag GmbH \& Co. KGaA, Weinheim chiral particles damage the mitochondrial membranes of cancer cells, thereby causing apoptosis, in a chiralitydependent manner whereby D-SPs have a stronger apoptosis-inducing effect than L-SPs (Fig. 8b) [9][9].

Tripathi et al. imparted chirality in carbon nanoparticles by controlled tethering of chiral molecules, i.e. TrÖger's base. It was found that chiral particles present groove-binding agents at the nanometer level, which can be positively enriching the cellular nucleus in MCF-7 breast cancer cells and give DNA enantiomer-specific recognition and help induce the apoptotic cascade of cancer cells. However, the negatively induced chiral carbon nanoparticles showed higher efficiency in inhibiting cell growth [72]. Schematic showing cancer cell apoptosis caused by the interaction between chiral carbon NPs and DNA (Fig. 9).

The above studies demonstrate that introducing chirality into a nanosystem can enhance its ability to evoke cancer cell apoptosis. In terms of underlying mechanism, the apoptosis-inducing effect of D-NPs is stronger than that of L-NPs due to the alkaline phosphatase secreted by cancer cells that dephosphorylates NP@L-pYs more rapidly than NP@D-pYs. The NP@L-pYs are mostly dephosphorylated before reaching the cancer cells. Therefore, there is no phosphate group and the NPs cannot bind to and kill the cell. However, the surface of NP@D-pYs is dephosphorylated at the cancer cells. This in-situ dephosphorization forms NP@D-Ys, which bind to cancer cells induces their apoptosis [71].

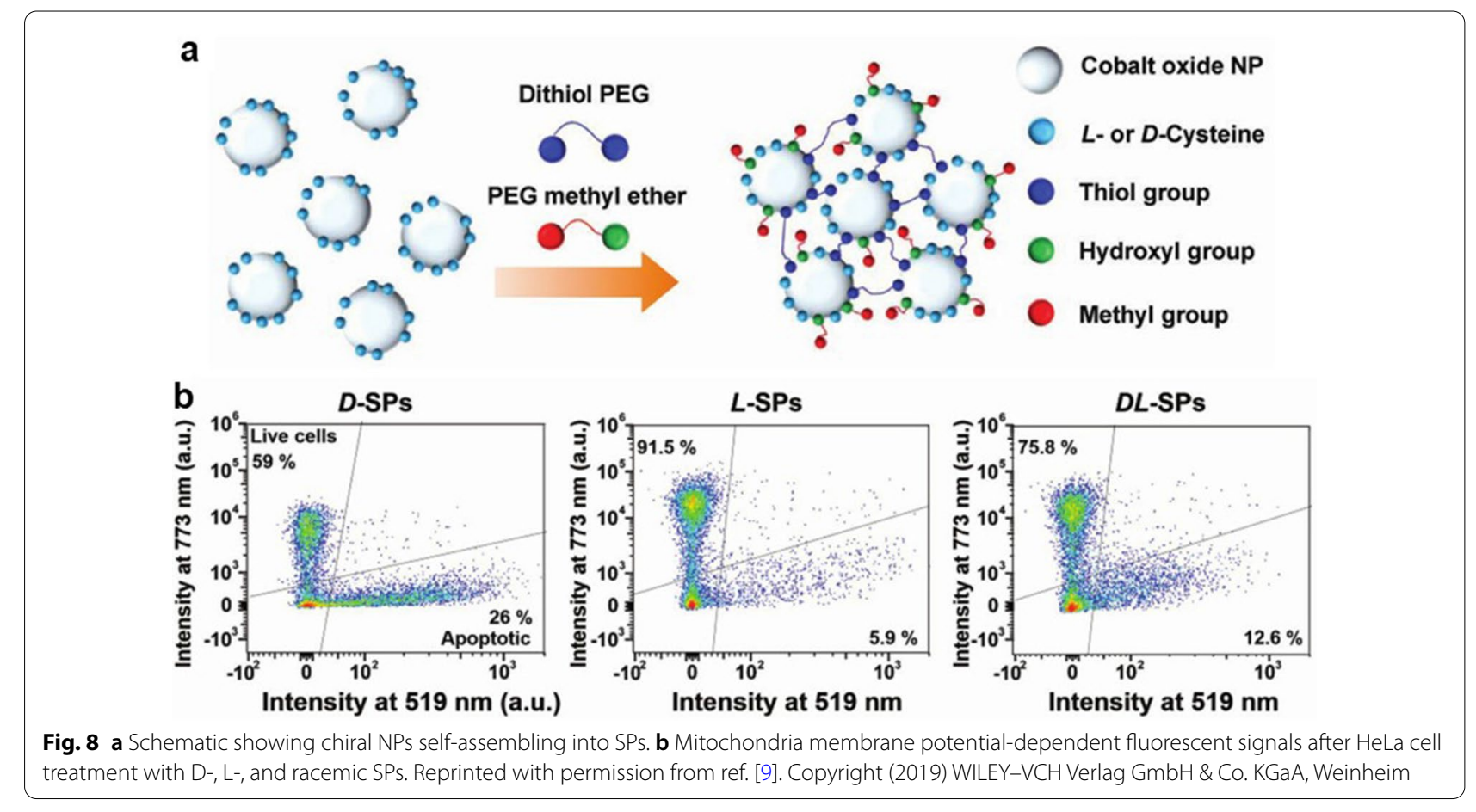




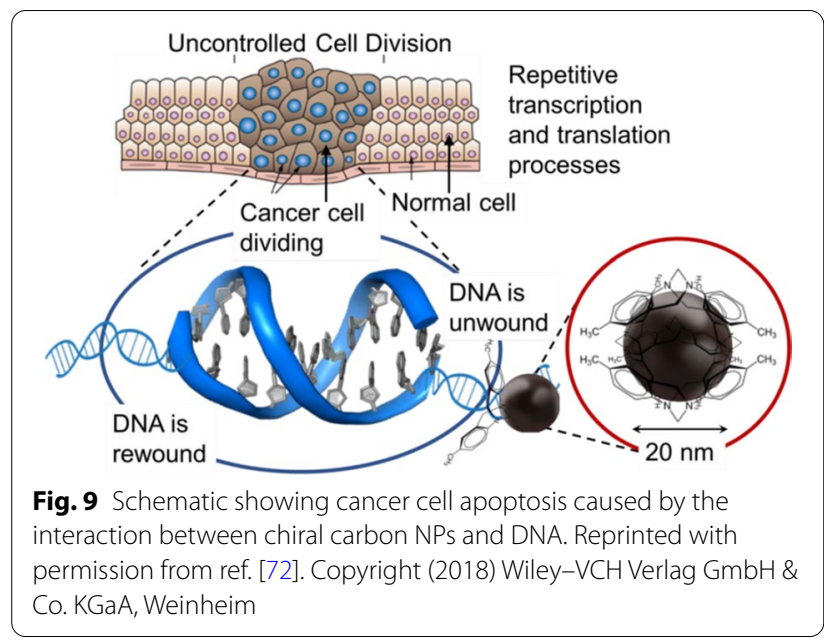

In addition, the D-chirality matches that of the L-phospholipid molecular layer on the surface of the cancer cell, leading to more efficient binding between them, and D-NPs are not easily degraded by natural proteases and coenzymes in the cellular environment, Both of these make D-NPs better absorbed by cancer cells and induce their apoptosis.Moreover, the efficacy of the negatively induced chiral carbon NPs in cancer treatment may due to the low intracellular $\mathrm{PH}$ value, the negatively induced chiral carbon NPs can more easily enter in nucleus by lipid-flipping thereby interacting with DNA in a grooved manner [72].

These results provide insights for the development of new chiral NPs that induce tumor cell apoptosis more effectively, thus realizing efficient targeted tumor therapy.

\section{Chiral nanomaterials for photothermal tumor therapy}

Photothermal therapy is a cancer-treatment strategy that involves local heat generation in cancer cells upon light radiation [73]. This technology uses NIR light to irradiate tumor tissues as it exhibits strong tissue penetration. Photothermal conversion then increases the temperature of the tumor tissue, thereby damaging tumor cells through protein denaturation and membrane disruption [74, 75]. In recent years, several studies have demonstrated that the heat generated by nano-photothermal materials not only has the effect of directly killing tumor cells, it can also inhibit tumor metastasis [76-78]. In addition, nanophotothermal materials can also be used in bioimaging, and they may also be modified in such a way as to impart properties that may be beneficial to chemotherapy, radiotherapy, and immunotherapy, making them effective multifunctional diagnostic and therapeutic agents against tumors $[79,80]$. Accordingly, the integration of diagnostic and therapeutic functions into single nanomaterials will realize the integration of nanomedicine in tumorimaging diagnosis and treatment.

Combining chirality with specific selectivity for biological systems and NPs with special size and surface properties-chiral NPs, which have more significant photothermal properties, and can be better applied to photothermal treatment of tumors [81-83]. For instance, Chen et al. introduced L-cysteine to the surface of molybdenum $\left(\mathrm{MoO}_{3}-\mathrm{x}\right)$ NPs with a large optical adsorption coefficient and successfully synthesized chiral molybdenum $\left(\mathrm{Cys}-\mathrm{MoO}_{3}-\mathrm{x}\right)$ and applied them to squamous cell carcinoma (OSCC) cells and found that they had a visible light (VL) to NIR dual PTT effect, with tumor cell fatality rates up to $89 \%$ [82]. Antaris et al. modified $(6,5)$ chiral SWCNTs with C18-PMH-mPEG to fabricate biocompatible SWCNTs. Chiral carbon nanotubes are highly biocompatible and therefore exhibit extended blood circulation and high tumor-uptake rates without losing their NIR photoluminescence (PL) properties. The SWCNTs were injected intravenously into a mouse tumor model and successfully used to heat the tumors photothermally to over $50{ }^{\circ} \mathrm{C}$ while simultaneously visualizing tumor accumulation through whole-animal NIRPL imaging [84]. Zhao et al. prepared HER2 aptamers of SK-BR-3 breast cancer cells and used them in the preparation of chiral Ag@Au core-shell NP assemblies (Ag@ Au CS NPs). The NPs were demonstrated to be stable, and they allowed sensitive and accurate detection of cancer cells that exhibit HER2 overexpression [85].

Yang et al. introduced N-isobutyryl-L(D)-cysteine enantiomers as stabilizers into $\mathrm{HgCl} 2$ and $\mathrm{Na} 2 \mathrm{~S}$ aqueous solutions as a means to synthesize chiral QDs ( $\beta$-HgS QDs). An aqueous $\beta-\mathrm{HgS}$ QD solution was heated to $50{ }^{\circ} \mathrm{C}$ upon laser irradiation, and the photothermal conversion efficiency was found to be stable over several cold-thermal cycles [59]. Li et al. used chiral cysteine as a reducing agent to reduce $\mathrm{MoO} 3$, obtaining a dual visible-light- and NIR-active nanomaterial (L/DCys-MO3-X NPs) with a strong chiral effect (Fig. 10). After being endocytosed by tumor cells, the NP generate temperatures of $40-50{ }^{\circ} \mathrm{C}$ in tumor cells upon absorbing visible- or NIR-CPL, thereby inducing cell death by denaturing intracellular proteins and rupturing cell membranes. L/D-Cys-MO3-X NPs exhibit higher photothermal conversion efficiency and tumor cell killing effect than the traditional hyperthermic agent molybdenum oxide. Notably, these chiral NPs show chiral selectivity for incident light. When irradiated by left-CPL, L-Cys-MoO3- $x$ has the highest efficiency for HeLa cell ablation in vitro, while the corresponding results are observed for D-Cys-MoO3-x and right-CPL [33]. Li Feng 


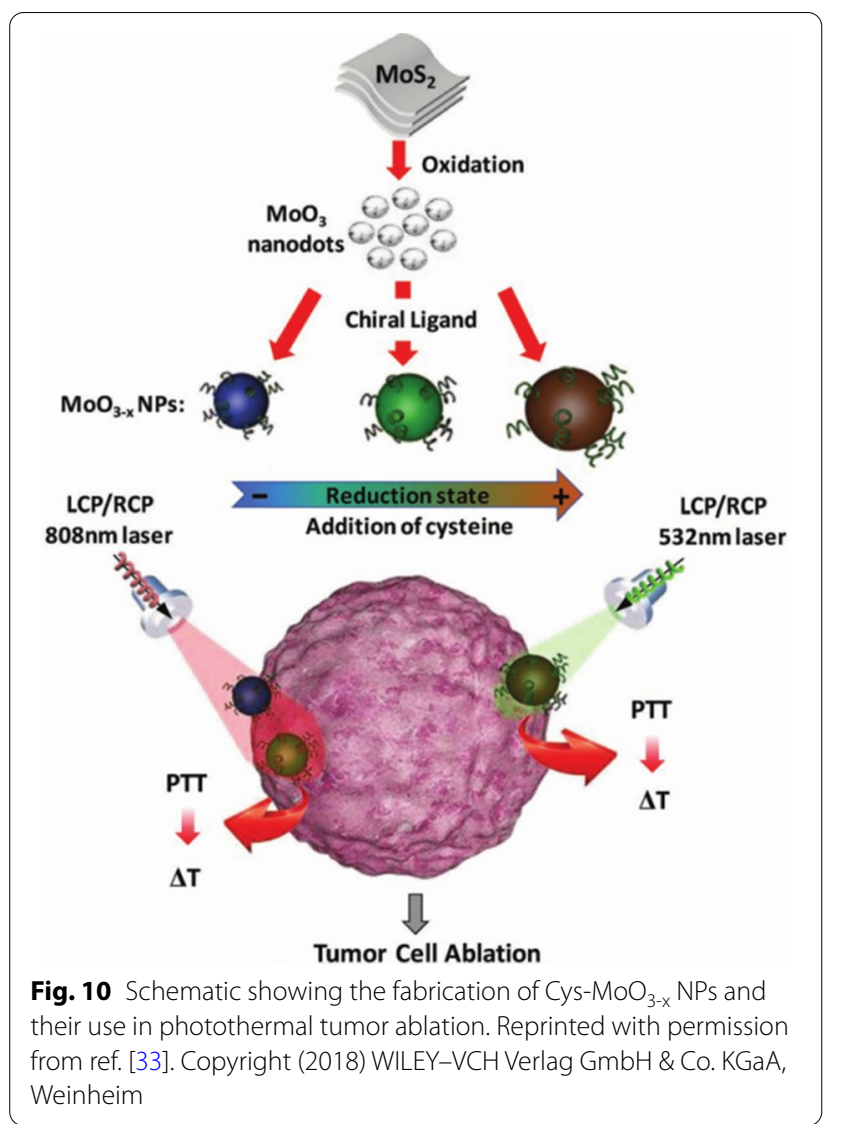

et al. synthesized chiral N-S-doped carbon dots in aqueous solution using cysteine, and their PL quantum yields were found to reach $41.26 \%$. Interestingly, the chiroptical properties and fluorescence intensity of the N-S-doped carbon dots are $\mathrm{pH}$ dependent. Additionally, L-carbon dots effect chirality-dependent glycolysis enhancement in T24 bladder cancer cells [86]. These studies demonstrate that chiral ligands can dramatically improve the optical properties of compounds, enabling their application to photothermal therapy and optical imaging of tumor cells.

In terms of mechanism, the introduction of chiral ligands improves the biocompatibility of a nanomaterial, increasing its blood-circulation half-life and thus uptake by tumor cells. Furthermore, the introduction of chiral ligands can improve the cancer-cell bioaffinity of a nanomaterial, strengthen its resistance to peptides and proteins in the blood, and endow it with chiral optical activity, NIR absorption, fluorescence emission, and photothermal conversion. There is a big chirality difference between L(or)D-NPs and Circulating tumor DNA (ctDNA). The influence of steric hindrance makes D-NPs more likely to bind to B-helix ctDNA than L-NPs, which may also be one of the reasons [87].
Based on the above studies, we believe that chiral recognition will come to play an important role in photothermal therapy. Based on the chiral activities, NIR fluorescence, biocompatibilities, chiral-recognition potential, and stable and efficient photothermal conversion, chiral nanomaterials are promising candidate agents for photothermal therapy and/or NIR fluorescent probes.

\section{Conclusions and future perspectives}

In summary, chiral nanomaterials can kill tumor cells and inhibit tumor growth by inducing cell apoptosis, autophagy, and photothermal ablation. The reasons as follows. Firstly, after the introduction of chiral amino acid ligands, a large number of amine groups and carboxyl groups are presented on the surfaces of the NPs, imparting excellent water solubility and suitable chemically reactive groups [29], which can greatly improve their biocompatible [51]; Secondly, Good endocytosis is the crucial first step for NPs accumulation in the cell. NPs as extracellular substance the main way to enter cells is micropinocytosis [47]. After enter the cell, the phospholipid layer on the surface of the cancer cell can combine with chiral NPs. Additionally, chiral NPs are intercalated between the G-C bases of circulating tumor DNA (ctDNA), thereby affecting the base stacking of ctDNA [88].

However, the autophagy, apoptosis, and photothermal ablation of tumor cells induced by chiral NPs appear to be D-chirality-dependent. In terms of potential mechanism: (1) due to the L-phospholipid molecular layer on the surface of the cancer cell is more likely to combine with heteroenantiomeric polymers (D-NPs), making D-NPs more easily absorbed and accumulated by cancer cells [52]. As D-NPs accumulate, more ATP will be produced by the reaction of NPs with biological macromolecules in the cell [4], and autophagy-related reactions depend on the expression of ATP $[56,57]$. D-NPs accumulate more, and the interaction with cell surface receptors induces more ROS [55], ROS are necessary for inducing autophagy [58]. (2) The biological activities and metabolism of endogenous proteins are regulated by chiralityspecific enzymes. Compared with L-NPs, D-NPs are not easily degraded by natural proteases and coenzymes in the cellular environment. It may be this biodegradation tolerance that allows them to continuously and more efficiently induce autophagy [33, 59]. (3) The alkaline phosphatase secreted by cancer cells dephosphorylates L-NPs faster than D-NPs. L-NPs are mostly dephosphorylated before reaching cancer cells. Therefore, without phosphate groups, L-NPs cannot bind to and kill cancer cells. However, the surface of D-NPs is dephosphorylated on cancer cells. This in situ dephosphorization method allows binding to cancer cells and inhibition of 
their growth [71]. (4) The intercalation binding between chiral-NP and G-C base of ctDNA is affected by steric hindrance, the binding ability of D-NPs and ctDNA is stronger than that for L-NPs, thereby inducing the death of cancer cells more efficiently [88]. Of course, there are exceptions. In the study of Li's group, the abundance of L-GSH and relative scarcity of D-GSH in biological systems lead to differences in the shell degradation, and ultimately in the autophagy induced by L-GSH-QDs being stronger than that by D-GSH-QDs[28]。

More interestingly, in some of the above researches, chiral NPs can discriminate model tumor cells from normal cells. This may be due to upon contact with the complex biological systems, the proteins will be progressively and selectively adsorbed on the NPs surface, which was defined as "protein corona" [89, 90]. Chiral NPs can specifically bind to tumor cell-related proteins, but have low affinity with normal cells [4]. An example is the high-affinity binding of chiral NPs to heat shock protein (HSP90) [54], which plays an important role in tumor progression [91]. Chiral NPs can interact strongly with transferrin [4], which is highly expressed in cancer cells [92] and shows high-affinity [93], to achieve tumor targeting [4]. In addition, the looser cytomembrane structure [94] and larger surface viscosity [95] tumor cells are more easily to be invaded as compared with the normal ones.

The commonality of their underlying mechanisms presents the possibility of developing nanomaterials that induce both tumor autophagy and apoptosis. In addition, the optical properties can also be significantly improved by introducing chiral ligands, allowing application to photothermal therapy and optical imaging of tumor cells. Thus, the synthesis of chiral nanopolymers may realize the combination of autophagy induction, apoptosis induction, photothermal therapy, and bioimaging.

However, despite the substantial achievements in the application of chiral nanomaterials to the field of tumor treatment, there remain issues and problems to be addressed. Firstly, most of the inorganic NPs reported are cadmium and/or gold based [28, 55]. These materials are either intrinsically biotoxic or non-biodegradable and therefore suboptimal for investigating chiral effects on nano-bio interactions. Furthermore, these materials have side effects in biological applications. Thus, to better explore the application of chiral nanomaterials in biomedical, a promising strategy is to utilize non-toxic, without side effects and degradable raw materials. Secondly, although they exhibit prominent advantages in terms of blood circulation and tumor accumulation, chiral nanomaterials are always subject to two major pharmacological deficiencies, i.e., poor proteolytic and high immunogenic stabilities, severely limiting their clinical application. It is very necessary to adjust the mechanical properties and degradation time of these chiral nanomaterials according to needs. The combined preparation of supramolecular complexes and polymers is an effective strategy to achieve enhanced stability, so its structure can be optimized and then reassembled to promote stability. Additionally, whether the use of high-power lasers for the stimulation of tumor cell death presents the risk of long-term radiation damage to healthy cells also is another critical issue. Therefore, the application of biomarkers to chiral nanomaterials may provide a clear boundary for nearinfrared light to irradiate tumor tissues, thereby minimizing the harmful effects of light on normal tissues.

Although this nanomaterials-based anti-tumor strategy is still at the laboratory stage of development and presents many challenges to be overcome, we believe that chiral nanomaterials show enormous promise and the potential to become a crucial tool in precision medicine.

\section{Acknowledgements}

The authors acknowledge the Department of Breast Surgery, Southwest Hospital, Army Medical University, and the Institute of Burn Research, Southwest Hospital, State Key Lab of Trauma, Burn and Combined Injury, Army Medical University.

\section{Authors' contributions}

The manuscript was written through contributions of all authors. All authors read and approved the final manuscript.

\section{Funding}

This work was supported in National Natural Science Foundation of China (NSFC) (No. 51703243), Chongqing Basic Research and Frontier Exploration Project (No. cstc2018jcyjA0137), Program of National Key Clinical Specialist Construction (No. 413F1Z113), Military Medical Staff Innovation Plan of Southwest Hospital (No. SWH2018BJLC-04) and Army Medical University (No. XZ-2019-505-042).

Availability of data and materials

Without restrictions.

\section{Declarations}

Ethics approval and consent to participate

Not applicable.

Consent for publication

All authors gave their consent for publication.

Competing interests

The authors declare that they have no competing interests.

Received: 7 May 2021 Accepted: 16 July 2021

Published online: 22 July 2021

References

1. Milton FP, Govan J, Mukhina MV, Gun'ko YK. The chiral nano-world: chiroptically active quantum nanostructures. Nanoscale Horiz. 2016;1(1):14-26.

2. Bird GH, Mazzola E, Opoku-Nsiah K, Lammert MA, Godes M, Neuberg DS, et al. Biophysical determinants for cellular uptake of hydrocarbon-stapled peptide helices. Nat Chem Biol. 2016;12(10):845-52. 
3. Rosenberg RA, Mishra D, Naaman R. Chiral selective chemistry induced by natural selection of spin-polarized electrons. Angew Chem Int Ed Engl. 2015;54(25):7295-8.

4. Wang X, Wang M, Lei R, Zhu SF, Zhao Y, Chen C. Chiral surface of nanoparticles determines the orientation of adsorbed transferrin and its interaction with receptors. ACS Nano. 2017;1 1(5):4606-16.

5. Pu F, Ren J, Qu X. Nucleobases, nucleosides, and nucleotides: versatile biomolecules for generating functional nanomaterials. Chem Soc Rev. 2018:47(4):1285-306.

6. Ishigami T, Suga K, Umakoshi H. Chiral recognition of L-amino acids on liposomes prepared with L-phospholipid. ACS Appl Mater Interfaces. 2015;7(38):21065-72.

7. Huang Y, Fu Y, Li M, Jiang D, Kutyreff CJ, Engle JW, et al. Chirality-driven transportation and oxidation prevention by chiral selenium nanoparticles. Angew Chem Int Ed Engl. 2020;59(11):4406-14.

8. Yeom J, Guimaraes PPG, Ahn HM, Jung BK, Hu Q, McHugh K, et al. Chiral supraparticles for controllable nanomedicine. Adv Mater. 2020;32(1): e1903878.

9. Tishigami T, Suga K, Umakoshi H. Chiral recognition of L-amino acids on liposomes prepared with L-phospholipid. ACS Appl Mater Interfaces. 2015;7(38):21065-72.

10. Hii JT, Duff HJ, Burgess ED. Clinical pharmacokinetics of propafenone. Clin Pharmacokinet. 1991;21(1):1-10

11. Soumerai SB, Avorn J, Gortmaker S, Hawley S. Effect of government and commercial warnings on reducing prescription misuse: the case of propoxyphene. Am J Public Health. 1988;77(12):1518-23.

12. Sohair $K$, Anthony P, Dagmara MG, Paul S. Segmental aging underlies the development of a parkinson phenotype in the AS/AGU rat. Cells. 2016;5(4):38.

13. Vargesson N. Thalidomide-induced teratogenesis: history and mechanisms. Birth Defects Res C Embryo Today Rev. 2015;105(2):140-56.

14. Chang G, Wieder BJ, Schindler F, Sanchez DS, Belopolski I, Huang SM, et al. Topological quantum properties of chiral crystals. Nat Mater. 2018;17(11):978-85.

15. Zhang $H$, He H, Jiang $X$, Xia Z, Wei W. Preparation and characterization of chiral transition-metal dichalcogenide quantum dots and their enantioselective catalysis. ACS Appl Mater Interfaces. 2018;10(36):30680-8.

16. Yang X, Gan L, Han L, Li D, Wang J, Wang E. Facile preparation of chiral penicillamine protected gold nanoclusters and their applications in cell imaging. Chem Commun. 2013:49(23):2302-4.

17. Marano F, Hussain S, Rodrigues-Lima F, Baeza-Squiban A, Boland S. Nanoparticles: molecular targets and cell signalling. Arch Toxicol. 2011;85(7):733-41.

18. Hao C, Xu L, Sun M, Zhang H, Kuang H, Xu C. Circularly polarized light triggers biosensing based on chiral assemblies. Chem Eur J. 2019;25(53):12235-40.

19. Masteri-Farahani M, Mollatayefeh N. Chiral colloidal CdSe quantum dots functionalized with cysteine molecules: new optical nanosensor for selective detection and measurement of morphine. Colloids Surf. 2019;596:78-84.

20. Song L, Wang S, Kotov NA, Xia Y. Nonexclusive fluorescent sensing for L/D enantiomers enabled by dynamic nanoparticle-nanorod assemblies. Anal Chem. 2012:84(17):7330-5.

21. Djunic I, Elezovic I, Marinkovic M, Suvajdzic-Vukovic N, Tomin D, Jankovic $\mathrm{G}$, et al. Osteolytic lesions marker in multiple myeloma. Med Oncol. 2011;28(1):237-40.

22. Martin HL, Smith L, Tomlinson DC. Multidrug-resistant breast cancer: current perspectives. Breast Cancer. 2014;6:1-13.

23. Coleman RE. Bone cancer in 2011: prevention and treatment of bone metastases. Nat Rev Clin Oncol. 2011;9(2):76-8.

24. Liu J, Detrembleur C, Grignard B, De Pauw-Gillet MC, Mornet S, TreguerDelapierre M, et al. Gold nanorods with phase-changing polymer corona for remotely near-infrared-triggered drug release. Chem Asian J. 2014;9(1):275-88

25. Liu J, Detrembleur C, Mornet S, Jérôme C, Duguet E. Design of hybrid nanovehicles for remotely triggered drug release: an overview. J Mater Chem B. 2015;3(30):6117-47.

26. Liu J, Detrembleur C, Debuigne A, De Pauw-Gillet MC, Mornet S, Vander Elst L, et al. Poly(acrylic acid)-block-poly(vinyl alcohol) anchored maghemite nanoparticles designed for multi-stimuli triggered drug release. Nanoscale. 2013;5(23):11464-77.
27. Li Y, Mei T, Han S, Han T, An F. Cathepsin B-responsive nanodrug delivery systems for precise diagnosis and targeted therapy of malignant tumors. Chin Chem Lett. 2020;31:3027-40.

28. Li Y, Zhou Y, Wang HY, Perrett S, Zhao Y, Tang Z, et al. Chirality of glutathione surface coating affects the cytotoxicity of quantum dots. Angew Chem Int Ed Engl. 2011;50(26):5860-4

29. Lim SY, Shen W, Gao Z. Carbon quantum dots and their applications. Chem Soc Rev. 2015;44(1):362-81.

30. Ru Y, Ai L, Jia T, Liu X, Yang B. Recent advances in chiral carbonized polymer dots: from synthesis and properties to applications. Nano Today. 2020;34: 100953.

31. Moloney MP, Gun'ko YK, Kelly JM. Chiral highly luminescent CdS quantum dots. Chem Commun. 2007:38:3900-2.

32. Nakashima T, Kobayashi Y, Kawai T. Optical activity and chiral memory of thiol-capped CdTe nanocrystals. J Am Chem Soc. 2009;131(30):10342-3.

33. Li Y, Miao Z, Shang Z, Cai Y, Xu X. A visible- and NIR-light responsive photothermal therapy agent by chirality-dependent MoO3x nanoparticles. Adv Funct Mater. 2020;30(4):1906311.

34. Mizushima N, Levine B, Cuervo AM, Klionsky DJ. Autophagy fights disease through cellular self-digestion. Nature. 2008:451(7182):1069-75.

35. Giansanti V, Torriglia A, Scovassi Al. Conversation between apoptosis and autophagy: "is it your turn or mine?" Apoptosis. 2011;16(4):321-33.

36. Deng S, Shanmugam MK, Kumar AP, Yap CT, Sethi G, Bishayee A. Targeting autophagy using natural compounds for cancer prevention and therapy. Cancer. 2019;125(8):1228-46.

37. Lin YC, Lin JF, Wen SI, Yang SC, Tsai TF, Chen HE, et al. Chloroquine and hydroxychloroquine inhibit bladder cancer cell growth by targeting basal autophagy and enhancing apoptosis. Kaohsiung J Med Sci. 2017;33(5):215-23.

38. Aita VM, Liang XH, Murty W, Pincus DL, Yu W, Cayanis E, et al. Cloning and genomic organization of beclin 1, a candidate tumor suppressor gene on chromosome 17q21. Genomics. 1999;59(1):59-65.

39. Zhou ZR, Yang ZZ, Wang SJ, Zhang L, Luo JR, Feng Y, et al. The Chk1 inhibitor MK-8776 increases the radiosensitivity of human triplenegative breast cancer by inhibiting autophagy. Acta Pharmacol Sin. 2017;38(4):513-23.

40. Kotcherlakota R, Rahaman ST, Patra CR. Nanomedicine for cancer therapy using autophagy: an overview. Curr Top Med Chem. 2018;18(30):2599-613.

41. Wei W, Rosenkrans ZT, Luo QY, Lan X, Cai W. Exploiting nanomaterial-mediated autophagy for cancer therapy. Small Methods. 2019:3(2):1800365.

42. Zheng W, Wei M, Li S, Le W. Nanomaterial-modulated autophagy: underlying mechanisms and functional consequences. Nanomedicine. 2016;11(11):1417-30

43. Peynshaert K, Manshian BB, Joris F, Braeckmans K, De Smedt SC, Demeester J, et al. Exploiting intrinsic nanoparticle toxicity: the pros and cons of nanoparticle-induced autophagy in biomedical research. Chem Rev. 2014;114(15):7581-609.

44. Deng J, Yao M, Gao C. Cytotoxicity of gold nanoparticles with different structures and surface-anchored chiral polymers. Acta Biomater. 2017:53:610-8.

45. Yao X, Hu Y, Cao B, Peng R, Ding J. Effects of surface molecular chirality on adhesion and differentiation of stem cells. Biomaterials. 2013;34(36):9001-9.

46. Deng J, Zheng H, Zheng X, Yao M, Li Z, Gao C. Gold nanoparticles with surface-anchored chiral poly(acryloyl-L(D)-valine) induce differential response on mesenchymal stem cell osteogenesis. Nano Res. 2016;9(12):3683-94.

47. Deng J, Wu S, Yao M, Gao C. Surface-anchored poly(acryloyl-L(D)-valine) with enhanced chirality-selective effect on cellular uptake of gold nanoparticles. Sci Rep. 2016;6:31595.

48. Liu GF, Zhang D, Feng CL. Control of three-dimensional cell adhesion by the chirality of nanofibers in hydrogels. Angew Chem Int Ed Engl. 2014;53(30):7789-93.

49. Wang $X$, Gan H, Sun T. Chiral design for polymeric biointerface: the influence of surface chirality on protein adsorption. Adv Funct. 2011;21(17):3276-81

50. Deng J, Li Z, Yao M, Gao C. Influence of albumin configuration by the chiral polymers-grafted gold nanoparticles. Langmuir. 2016;32(22):5608-16. 
51. Hu C, Xu X, Zhang X, Li Y, Li Y, Gu Z. Bioinspired design of stereospecific d-protein nanomimics for high-efficiency autophagy induction. Chem Mater. 2017;29:7658-62.

52. Sun M, Hao T, Li X, Qu A, Xu L, Hao C, et al. Direct observation of selective autophagy induction in cells and tissues by self-assembled chiral nanodevice. Nat Commun. 2018;9(1):4494.

53. Wang Y, Xia Y. Near-infrared optically active CuS nanocrystals: sacrificial template-ligand exchange integration fabrication and chirality dependent autophagy effects. J Mater Chem B. 2020;8(35):7921-30.

54. Xin Q, Zhang H, Liu Q, Dong Z, Xiang H, Gong JR. Extracellular biocoordinated zinc nanofibers inhibit malignant characteristics of cancer cell. Nano Lett. 2015;15(10):6490-3.

55. Yuan L, Zhang F, Qi X, Yang Y, Yan C, Jiang J, et al. Chiral polymer modified nanoparticles selectively induce autophagy of cancer cells for tumor ablation. J Nanobiotechnol. 2018;16(1):55.

56. Li FJ, Xu ZS, Soo AD, Lun ZR, He CY. ATP-driven and AMPK-independent autophagy in an early branching eukaryotic parasite. Autophagy. 2017;13(4):715-29.

57. Martin S, Dudek-Peric AM, Garg AD, Roose H, Demirsoy S, Van Eygen $S$, et al. An autophagy-driven pathway of ATP secretion supports the aggressive phenotype of BRAF(V600E) inhibitor-resistant metastatic melanoma cells. Autophagy. 2017;13(9):1512-27.

58. Ding F, Li Y, Liu J, Liu L, Yu W, Wang Z, et al. Overendocytosis of gold nanoparticles increases autophagy and apoptosis in hypoxic human renal proximal tubular cells. Int J Nanomed. 2014;9:4317-30.

59. Yang F, Gao G, Wang J, Chen R, Zhu W, Wang L, et al. Chiral $\beta$-HgS quantum dots: aqueous synthesis, optical properties and cytocompatibility. J Colloid Interface Sci. 2019;537:422-30.

60. Folkers H, Guy G, Richard. Pharmacokinetic optimization in drug research: biological, physicochemical, and computational strategies. Lipophilicity Symposium. 2007.

61. Chang B, Zhang M, Qing G, Sun T. Dynamic biointerfaces: from recognition to function. Small. 2015;11(9-10):1097-112.

62. Zhang M, Qing G, Sun T. Chiral biointerface materials. Chem Soc Rev. 2012:41(5):1972-84.

63. Gammon ST, Villalobos VM, Prior JL, Sharma V, Piwnica-Worms D. Quantitative analysis of permeation peptide complexes labeled with Technetium-99m: chiral and sequence-specific effects on net cell uptake. Bioconjug Chem. 2003;14(2):368-76.

64. Kerr JF, Wyllie AH, Currie AR. Apoptosis: a basic biological phenomenon with wide-ranging implications in tissue kinetics. Br J Cancer. 1972;26(4):239-57.

65. Zhirnov OP, Konakova TE, WolffT, Klenk HD. NS1 protein of influenza A virus down-regulates apoptosis. J Virol. 2002;76(4):1617-25.

66. Yao J, Qian CJ, Ye B, Zhang X, Liang Y. ERK inhibition enhances TSAinduced gastric cancer cell apoptosis via NF-KB-dependent and Notchindependent mechanism. Life Sci. 2012;91(5-6):186-93.

67. Dunn GP, Bruce AT, Ikeda H, Old LJ, Schreiber RD. Cancer immunoediting: from immunosurveillance to tumor escape. Nat Immunol. 2002;3(11):991-8.

68. Shaniba VS, Aziz AA, Jayasree PR, Kumar PRM, Manilkara Zapota LP. Royen leaf extract derived silver nanoparticles induce apoptosis in human colorectal carcinoma cells without affecting human lymphocytes or erythrocytes. Biol Trace Elem Res. 2019;192(2):160-74.

69. Halawani EM, Hassan AM, Gad El-Rab SMF. Nanoformulation of biogenic cefotaxime-conjugated-silver nanoparticles for enhanced antibacterial efficacy against multidrug-resistant bacteria and anticancer studies. Int J Nanomed. 2020;15:1889-901.

70. Zada S, Ahmad A, Khan S, Yu X, Chang K, lqbal A, et al. Biogenic synthesis of silver nanoparticles using extracts of Leptolyngbya JSC-1 that induce apoptosis in HeLa cell line and exterminate pathogenic bacteria. Artif Cells Nanomed Biotechnol. 2018;46(sup3):S471-s480.

71. Du X, Zhou J, Wang J, Zhou R, Xu B. Chirality controls reaction-diffusion of nanoparticles for inhibiting cancer cells. ChemNanoMat. 2017;3(1):17-21.

72. Tripathi I, Misra SK, Ostadhossein F, Srivastava I, Pan D. Synthesis of chiral carbo-nanotweezers for enantiospecific recognition and DNA duplex winding in cancer cells. ACS Appl Mater Interfaces. 2018;10(44):37886-97.

73. Shibu ES, Hamada M, Murase N, Biju V. Nanomaterials formulations for photothermal and photodynamic therapy of cancer. J Photochem Photobiol C. 2013;15:53-72
74. Jaque D, Martínez Maestro L, Del Rosal B, Haro-Gonzalez P, Benayas A, Plaza JL, et al. Nanoparticles for photothermal therapies. Nanoscale. 2014;6(16):9494-530.

75. Zeng X, Xiao Y, Lin J, Li S, Zhou H, Nong J, et al. Near-infrared II dye-protein complex for biomedical imaging and imaging-guided photothermal therapy. Adv Healthc Mater. 2018;7(18):1800589.

76. Jiang Q, Luo Z, Men Y, Yang P, Peng H, Guo R, et al. Red blood cell membrane-camouflaged melanin nanoparticles for enhanced photothermal therapy. Biomaterials. 2017;143:29-45.

77. Chen HY, Deng J, Wang Y, Wu CQ, Li X, Dai HW. Hybrid cell membranecoated nanoparticles: a multifunctional biomimetic platform for cancer diagnosis and therapy. Acta Biomater. 2020;112:1-13.

78. Chen Y, Shen X, Han S, Wang T, Zhao J, He Y, et al. Irradiation pretreatment enhances the therapeutic efficacy of platelet-membrane-camouflaged antitumor nanoparticles. J Nanobiotechnol. 2020;18(1):101.

79. Müller J, Wunder A, Licha K. Optical imaging. Recent Results Cancer Res. 2013;187:221-46

80. An F, Yang Z, Zheng M, Mei T, Deng G, Guo P, et al. Rationally assembled albumin/indocyanine green nanocomplex for enhanced tumor imaging to guide photothermal therapy. J Nanobiotechnol. 2020;18(1):49.

81. Lee HE, Ahn HY, Mun J, Lee YY, Kim M, Cho NH, et al. Amino-acid- and peptide-directed synthesis of chiral plasmonic gold nanoparticles. Nature. 2018;556(7701):360-5.

82. Chen J, Li Q, Wang F, Yang M, Xie L, Zeng X. Biosafety, nontoxic nanoparticles for VL-NIR photothermal therapy against oral squamous cell carcinoma. ACS Omega. 2021;6(17):11240-7.

83. Fukuda R, Umeyama T, Tsujimoto M, Ishidate F, Murakami T. Sustained photodynamic effect of single chirality-enriched single-walled carbon nanotubes. Carbon. 2020;161:718-25.

84. Antaris AL, Robinson JT, Yaghi OK, Hong G, Diao S, Luong R, et al. Ultralow doses of chirality sorted $(6,5)$ carbon nanotubes for simultaneous tumor imaging and photothermal therapy. ACS Nano. 2013;7(4):3644-52.

85. Zhao Y, Yang Y, Zhao J, Weng P, Pang Q, Song Q. Dynamic chiral nanoparticle assemblies and specific chiroplasmonic analysis of cancer cells. Adv Mater. 2016;28(24):4877-83

86. Li F, Li Y, Yang X, Han X, Jiao Y, Wei T, et al. Highly fluorescent chiral N-Sdoped carbon dots from cysteine: affecting cellular energy metabolism. Angew Chem Int Ed Engl. 2018;57(9):2377-82.

87. Suzuki N, Wang Y, Elvati P, Qu ZB, Kim K, Jiang S. Chiral graphene quantum dots. ACS Nano. 2016;10(2):1744-55.

88. Li F, Li S, Guo X, Dong Y, Yao C, Liu Y, et al. Chiral carbon dots mimicking topoisomerase I to mediate the topological rearrangement of supercoiled DNA enantioselectively. Angew Chem Int Ed Engl. 2020;59(27):11087-92.

89. Deng J, Dahai YU, Gao CY. Biological identity of nanomaterials: opportunities and challenges. Sci China Chem. 2013;56(011):1533-41.

90. Mahmoudi M, Bertrand N, Zope H, Farokhzad OC. Emerging understanding of the protein corona at the nano-bio interfaces. Nano Today. 2016;11(6):817-32

91. Chen JS, Hsu YM, Chen CC, Chen LL, Lee CC, Huang TS. Secreted heat shock protein 90alpha induces colorectal cancer cell invasion through CD91/LRP-1 and NF-kappaB-mediated integrin alphaV expression. J Biol Chem. 2010:285(33):25458-66.

92. Prost AC, Ménégaux F, Langlois P, Vidal JM, Koulibaly M, Jost JL, et al. Differential transferrin receptor density in human colorectal cancer: a potential probe for diagnosis and therapy. Int J Oncol. 1998;13(4):871-5.

93. Vyas SP, Sihorkar V. Endogenous carriers and ligands in non-immunogenic site-specific drug delivery. Adv Drug Deliv Rev. 2000;43(2-3):101-64.

94. Allen TD, lype PT. The surface morphology of normal and malignant rat liver epithelial cells in culture. Vitro. 1976;12(12):837-44.

95. Chattoraj S, Bhattacharyya K. Fluorescent gold nanocluster inside a live breast cell: etching and higher uptake in cancer cell. J Phys Chem C. 2015;118(38):22339-46

\section{Publisher's Note}

Springer Nature remains neutral with regard to jurisdictional claims in published maps and institutional affiliations. 